\title{
A systematic review of dietary interventions for gestational weight gain and gestational diabetes in overweight and obese pregnant women
}

\author{
Reeta Lamminpää $^{1}$ Katri Vehviläinen-Julkunen ${ }^{2}$ Ursula Schwab ${ }^{3,4}$
}

Received: 25 April 2017 / Accepted: 13 October 2017 / Published online: 11 November 2017

(c) The Author(s) 2017. This article is an open access publication

\begin{abstract}
Purpose The number of overweight and obese women is increasing in the obstetric population. The aim of this study was to review studies that reported results related to the efficacy of dietary interventions on gestational weight gain (GWG) or the prevention of gestational diabetes (GDM) in overweight and obese women.

Methods The search was performed using the CINAHL, PubMed, Scopus and Medic electronic databases and limited to the years between 2000 and March 2016. This systematic review includes 15 research articles of which 12 were randomized controlled trials, and three were controlled trials. Three main categories emerged as follows: (1) the types of interventions, (2) the contents of the interventions and (3) the efficacy of the intervention on GWG and the prevention of GDM. The quality of the selected studies was evaluated using the AHRQ Methods Reference Guide for Effectiveness and Comparative Effectiveness Reviews.

Results Of the selected 15 studies, eight included a specified diet with limited amounts of nutrients or energy, and the others included a dietary component along with other
\end{abstract}

Reeta Lamminpää

reeta.lampinen@uef.fi

1 Department of Nursing Science, Faculty of Health Sciences, University of Eastern Finland, P.O. BOX 1627, 70211 Kuopio, Finland

2 Department of Nursing Science, Faculty of Health Sciences, University of Eastern Finland and Kuopio University Hospital, 70029 Kuopio, Finland

3 Institute of Public Health and Clinical Nutrition, Faculty of Health Sciences, University of Eastern Finland, 70211 Kuopio, Finland

4 Institute of Clinical Medicine, Internal Medicine, Kuopio University Hospital, 70029 Kuopio, Finland components. Ten studies reported significant differences in the measured outcomes regarding GWG or the prevention of GDM between the intervention and the control groups. Conclusions This review confirms the variability in the strategies used to deliver dietary interventions in studies aiming to limit GWG and prevent GDM in overweight and obese women. Inconsistency in the provider as well as the content of the dietary interventions leaves the difficulty of summarizing the components of effective dietary interventions.

Keywords Dietary intervention - Gestational weight gain $\cdot$ Gestational diabetes $\cdot$ Systematic review

\section{Introduction}

Obesity is a public health concern in Finland and other developed countries. Overweight is defined as a body mass index $(\mathrm{BMI}) \geq 25$, and obesity is defined as a BMI $\geq 30$ [1]. Generally, in European countries, the percentages of obese, pregnant women vary from $7 \%$, in Poland, to $21 \%$, in Scotland. In Norway, Sweden, Malta and Denmark, the percentages were $12 \%$ in 2010 , which were quite similar to those in Finland [2]. In 2015, in Finland, the average pre-pregnancy BMI of women giving birth was $24.6 \mathrm{~kg} / \mathrm{m}^{2}$, and every third woman giving birth was categorized as overweight, while $13 \%$ were categorized as obese [3].

Overweight and obesity can lead to significant medical problems during pregnancy [4]. These conditions are associated with numerous maternal, foetal and neonatal complications [5, 6]. Overweight and obese women have an increased risk of gestational hypertension, preeclampsia and gestational diabetes (GDM) $[4,7,8]$. For the foetus, the risks caused by maternal overweight and obesity include 
childhood obesity, insulin resistance, and hyperlipidaemia [9]. Excessive weight gain during pregnancy is also associated with a greater newborn birthweight and a risk of childhood overweight and obesity [10].

GDM is defined as an abnormal glucose tolerance that develops or is recognized in pregnancy. The definition includes those women with previously undiagnosed diabetes and those with pregnancy-induced high glucose levels. The diagnosis is based on glucose levels in a $75 \mathrm{~g}$ oral glucose tolerance test (OGTT). In Finland, the proportion of women with GDM has increased rapidly during the past years from $10 \%$ in 2008 to $16 \%$ in 2015 [3].

For overweight and obese women, the recommended weight gain is less than that for normal weight women, which is $7-11.5 \mathrm{~kg}$ for those with a BMI of $25-29.9$ and only $5-9 \mathrm{~kg}$ for those with a BMI of 30 or above [11]. It has been shown that women who can limit their gestational weight gain (GWG) are also more likely to show less weight retention after pregnancy [12]. Ideally, obese women should be advised and motivated to maintain a healthy diet, exercise and manage their weight even at pre-conception and throughout the pregnancy [13]. Because overweight and obesity are associated with numerous risks during pregnancy, it would be ideal to prevent the risks by losing weight pre-conceptionally. However, this is difficult to achieve; thus, lifestyle interventions are needed [9].

Reviews related to the impact of different interventions on overweight and obese pregnant women have been conducted [14-20]. A perspective of these previous reviews shows the wide range of the effectiveness of the interventions on GWG, energy intake during pregnancy, pregnancy outcomes and prevention of GDM. The studies included in these reviews also range from dietary interventions to lifestyle and exercise interventions. The results of these studies on overweight and obese pregnant women reflect the variability in the impact of the interventions and the quality of the studies, and, therefore, implications for future studies or practice are not consistent. A systematic review by Flynn and colleagues [21] focused on the effective approaches in intervention studies $(n=13)$ that aimed to reduce obesity in pregnancy. The results confirmed the difficulty of developing clinical guidelines based on the large methodological variability in the previous studies. However, there appears to be evidence that diet-based interventions are effective in controlling GWG and reducing the adverse pregnancy outcomes as compared with other methods [15, 21, 22]. A meta-analysis by Rogozinska et al. (2016) managed to identify the components, including GDM, of maternal composite outcomes that are required for assessment of the diet and lifestyle interventions in pregnant women [23]. To the best of our knowledge, only a few reviews have focused on interventions targeted for overweight and obese pregnant women aiming to prevent GDM and do not report results focused on specifically the role of dietary interventions but include other interventions as well, which makes it difficult to conclude the effect of dietary components. In this review, the interventions are limited with dietary interventions or interventions including a dietary component to explore the efficacy of diet-based interventions on overweight and obese pregnant women.

It is important to identify the effective dietary components in the interventions that are effective in GWG or the prevention of GDM in an overweight and obese pregnant population. The aim of this systematic literature review was to answer the following research question: "What are the strengths and weaknesses of dietary intervention studies focusing on GWG and the prevention of GDM?" The aim was to describe the efficacy of dietary interventionstargeted for overweight and obese pregnant women-that limit GWG and prevent GDM. The PICOS criteria for the inclusion of studies included population, i.e., overweight $(B M I \geq 25)$ or obese $(B M I \geq 30)$ pregnant women; intervention, i.e., whether a dietary intervention or a dietary component was included; comparisons, i.e., no intervention or intervention with a differing content; outcomes, i.e., GWG and/or GDM; and study design, i.e., randomized controlled trials or controlled trials.

\section{Methods}

\section{Literature search}

The literature search was performed electronically between February and March 2016 (the date of the last search was 11/3/2016). The selected electronic databases were CINAHL, PubMed, Scopus and Medic. The following search strategy was used (example in CINAHL): gestational OR pregnan* AND "weight gain" OR diabetes AND overweight OR obes* AND intervent*. The search terms were formulated with the help of a university librarian. The search was limited to publications between the years 2000 and 2016, and the results were limited to "Peer-Reviewed" and "English language".

\section{Inclusion and exclusion criteria}

The inclusion and exclusion criteria are shown in Table 1.

\section{Search results and data evaluation}

First, the electronic search resulted in a total of 2268 titles, of which 1743 were identified after excluding duplicates. Altogether, 1743 titles were screened, and irrelevant titles were excluded, leaving 340 titles for careful screening based on the abstracts. The screening of the abstracts 
Table 1 The inclusion and exclusion criteria

\begin{tabular}{ll}
\hline Study inclusion criteria & Study exclusion criteria \\
\hline Studies with overweight or obese pregnant women as a target group & $\begin{array}{c}\text { Studies with an inadequate description of the dietary component used in } \\
\text { the intervention }\end{array}$ \\
$\begin{array}{l}\text { Studies with a dietary intervention or an intervention including a } \\
\text { dietary component }\end{array}$ & $\begin{array}{c}\text { Studies with only an exercise-based intervention without any dietary } \\
\text { component }\end{array}$ \\
Studies with outcomes including GWG or GDM & $\begin{array}{l}\text { Studies mainly focused on measuring GWG in the postpartum period } \\
\text { Studies written in English }\end{array}$ \\
Studies with a randomized controlled trial or controlled trial design & Studies with small sample sizes, $n<40$ \\
\hline
\end{tabular}

resulted in 70 remaining research articles that were subjected to a full-text examination. Of these 70 articles, 53 were excluded because they did not meet the inclusion criteria. In total, 17 articles were included in the quality assessment (Fig. 1) The quality assessment was performed using the AHRQ Methods Reference Guide for
Effectiveness and Comparative Effectiveness Reviews quality assessment tool [24]. The quality assessment was performed with the cooperation of three researchers. Based on the quality assessment, six studies were graded as $\mathrm{A}$; nine were graded as $\mathrm{B}$; and two were graded as $\mathrm{C}$. Those studies that were graded as $\mathrm{C}$ were excluded from
Fig. 1 Flowchart presenting the main phases of the literature search process

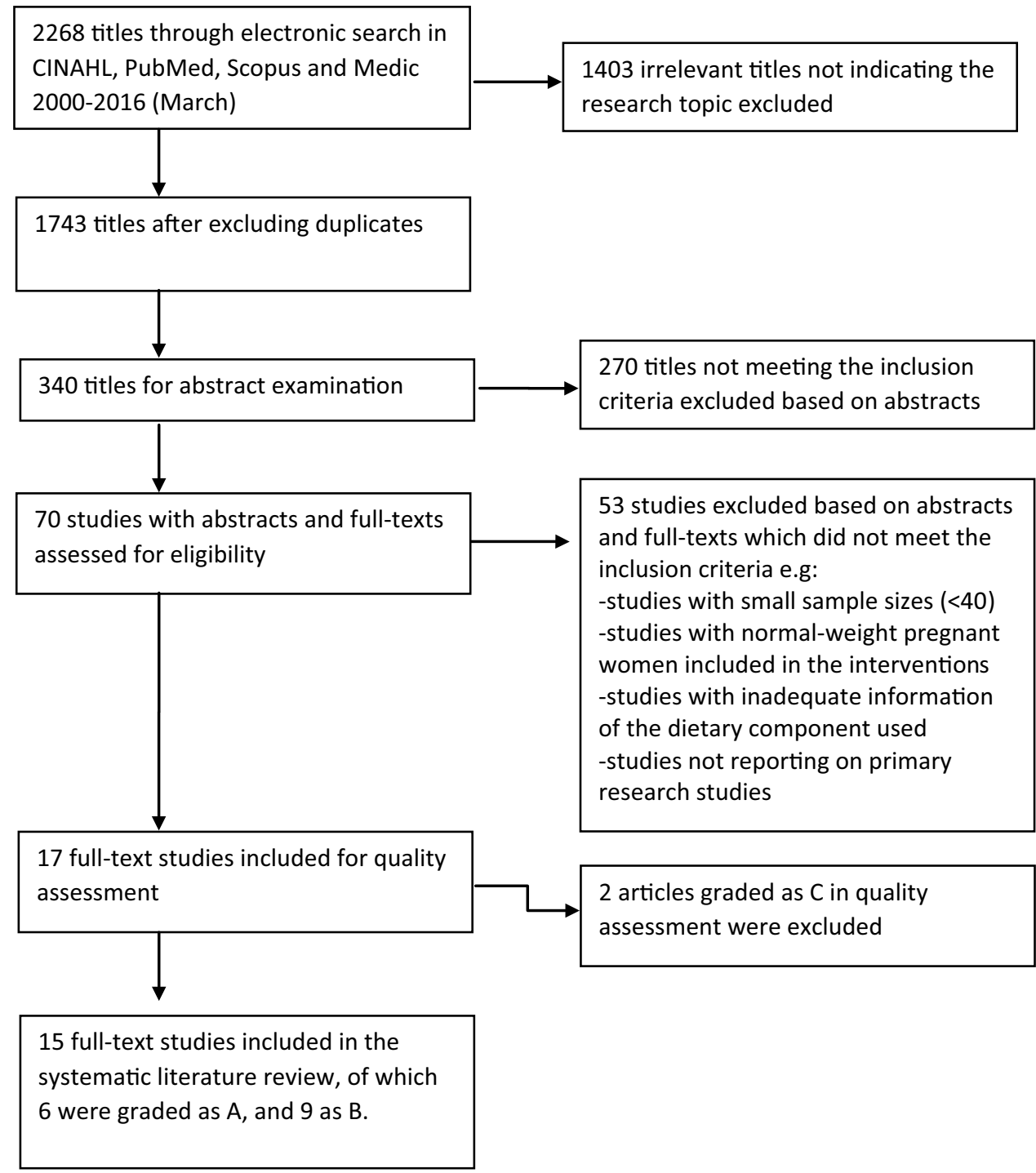


the review to reduce the risk of bias at both the study and outcome levels.

Figure 1.

\section{Analysis of the data}

The synthesis in this review was performed by analyzing the 15 selected research articles regarding dietary interventions focused on GWG and the prevention of GDM in overweight and obese women. After tabulating the studies, the results were synthesized using the content analysis method [25].

\section{Results}

\section{Study characteristics}

Of the 15 studies selected for this systematic review, 12 were randomized controlled trial (RCT) studies, and 3 were controlled trial (CT) studies.

The impact of the intervention was assessed on both the GDM and GWG in 4 studies [26-29], the GDM in 1 study [30], and the GWG in 10 studies [12, 31-39]. The majority of the studies were conducted in Australia $(20 \% n=3)$, USA $(20 \% n=3)$ and Denmark $(20 \% n=3)$. The sample sizes had a wide range from 50 to 1555 . Mostly, the overweight and obese pregnant women were recruited to the intervention before the 20th gestational week ( $n=10$ studies) or during the 1st trimester of pregnancy ( $N=5$ studies). Six studies [30-34] used food records/diary as the dietary assessment method; two studies used a Food Frequency Questionnaire (FFQ) [28, 29]; and two studies used 24-hour recall [30, 37]. Two studies $[12,27]$ used $\log$ s for the women to report their food consumption, and an audit of items consumed was used in one study [26]. Detailed information regarding the study characteristics is shown in Table 2.

\section{The types of interventions}

The approach of the dietary interventions varied among the 15 selected studies. Seven studies [27, 28, 32, 34-37] referred to "Lifestyle interventions", four [30, 31, 33, 39] studies referred to "Dietary interventions" and the remaining studies included different variations in defining the type of intervention, but all studies included a dietary component. Eight studies [28, 30-34, 36, 39] included a specified diet with limited amounts of allowed nutrients or energy in the dietary intervention. The remaining seven studies used a strategy including dietary advice or counselling related to healthy eating and a healthy diet during pregnancy mostly based on the National Nutritional Recommendations.

The counselling was provided by a nutritionist or a dietitian in most of the studies [12, 28, 30, 31, 33-36, 39], but in some cases, the counselling was provided by another health care provider, such as a health coach or a lifestyle coach, alone or accompanied by a dietitian or a nutritionist $[12,27$, 29, 32, 36-38]. Midwife-led interventions were rare. The counselling was provided individually in seven studies [26, $27,30,35,37-39]$, in groups in three studies [30, 31, 33] and both individually and in groups in three studies [12, 29, $33]$. In two studies [28, 36], the mode of delivering advice was not clearly described. In addition to the individual or group sessions providing dietary advice or the counselling, some of the studies reported giving written material for the women to support the process, such as handbooks, booklets or guides, containing information related to healthy food, nutrition [29, 30, 37, 38] and benefits to avoiding excessive weight gain [38].

Exercise or physical activity was included in 10 studies [12, 27-29, 33, 35-39] as a component of the intervention. The role of physical activity varied from the mildest level of encouraging pregnant women to engage in physical activity during the intervention to interventions in which exercise and diet were combined.

\section{The content of the dietary interventions}

The content of the diets with limited amounts of protein, fat and carbohydrate intake used in the dietary interventions are described in detail in Table 2. One study limited the energy intake to $1700 \mathrm{kcal} /$ day for overweight pregnant women and $1800 \mathrm{kcal} /$ day for obese pregnant women [28]. Another study used the pattern of total energy intake per day based on weight as $25 \mathrm{kcal} / \mathrm{kg}$ for overweight women and $20 \mathrm{kcal} /$ $\mathrm{kg}$ for obese women [36]. The Dietary Approaches to Stop Hypertension (DASH) dietary pattern with individualized calculated daily calorie goals was used in one study [33], and another study referred to a Mediterranean style, hypocaloric diet [39].

\section{The effectiveness of the dietary interventions in relation to GWG and GDM}

Of the total 15 studies, 10 studies reported significant differences between the study and the control groups in limiting the GWG $(n=10)$ and/or preventing GDM $(n=3)$, of which seven focused on GWG, and three focused on both.

The remaining five studies [29, 30, 34, 37, 39] reported no significant differences between the intervention and the control groups. The similarities among the effective interventions were a combination of a dietary component and a certain extent of physical activity. However, there were three studies $[26,31,32]$ with dietary interventions or interventions with a dietary component that were effective without physical activity. The effective interventions consisted of an inclusion of a dietitian or a nutritionist, but the interventions 


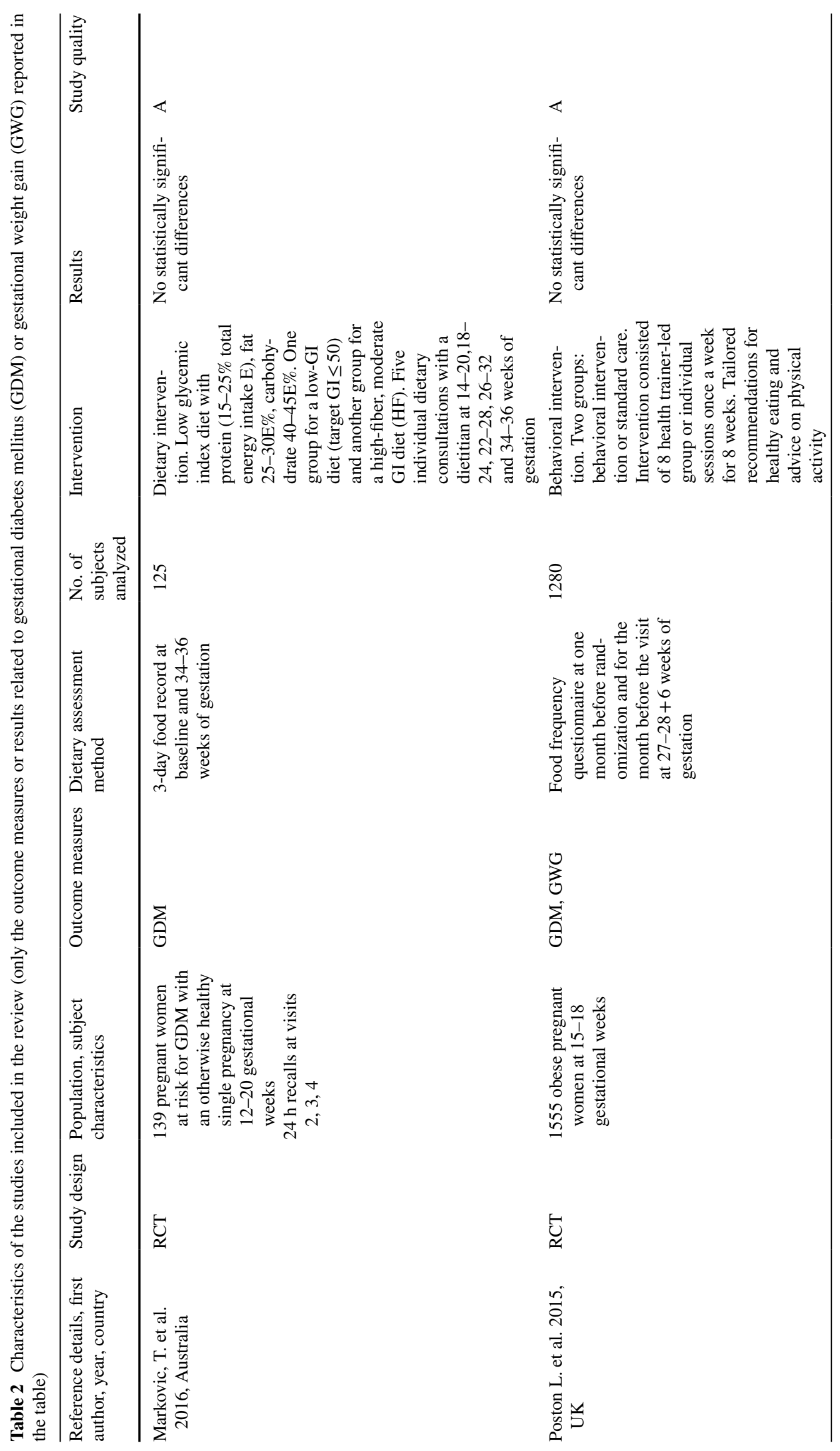




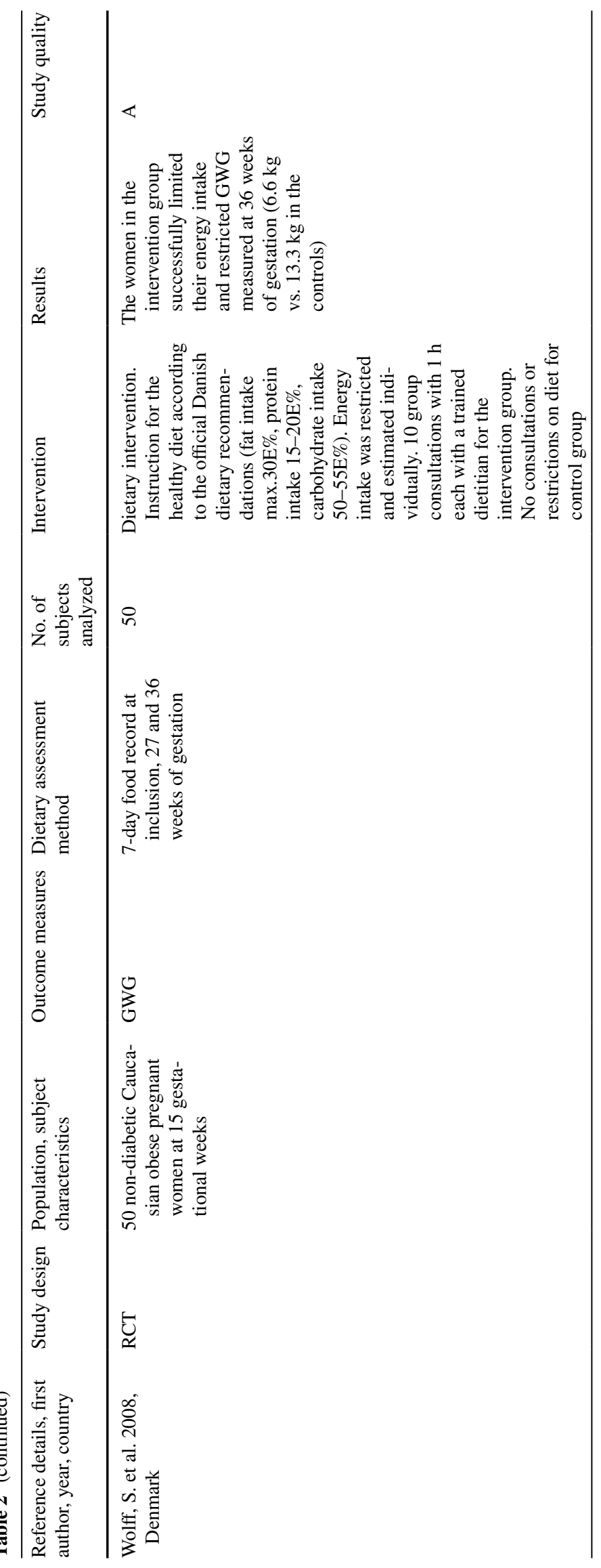




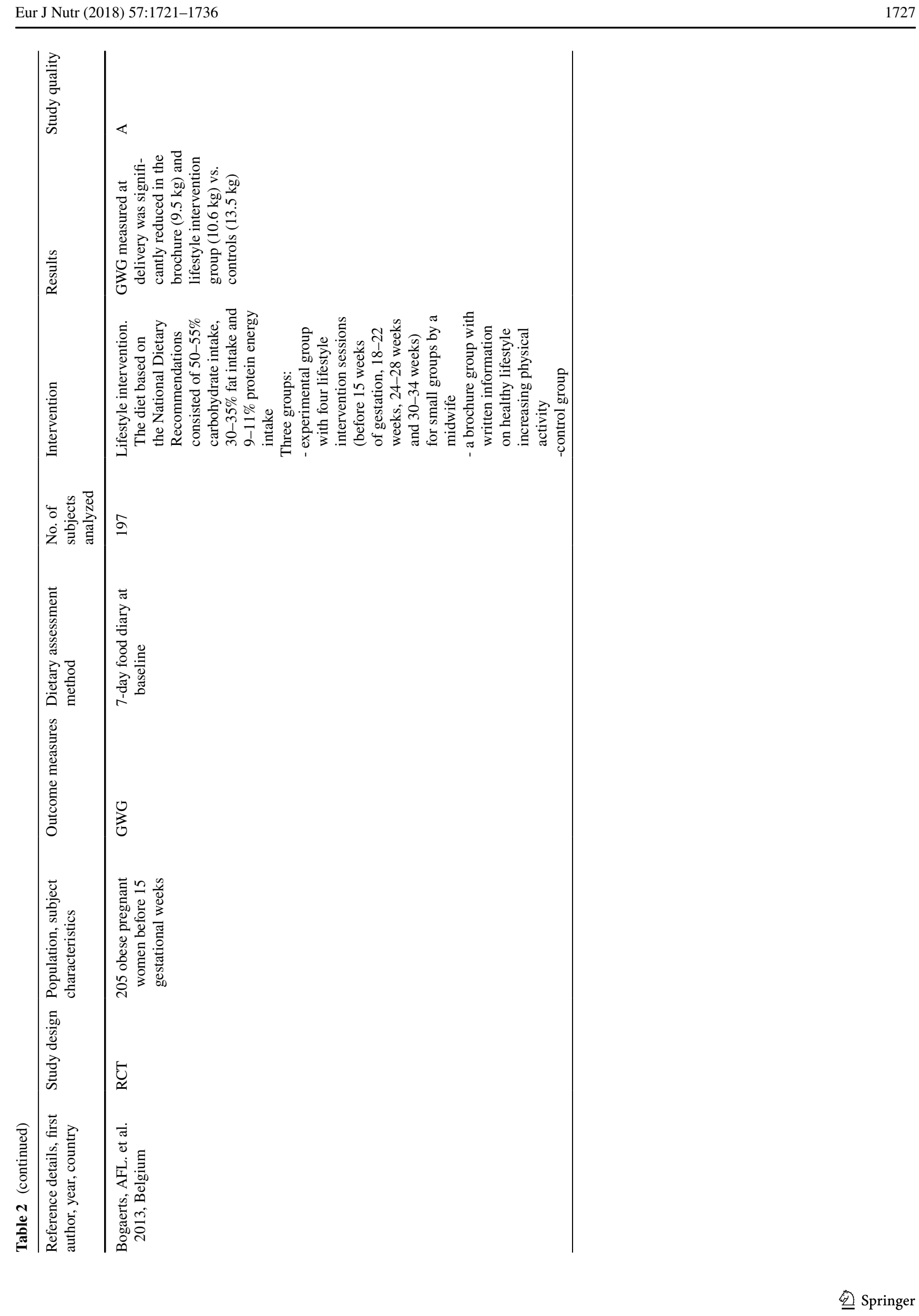




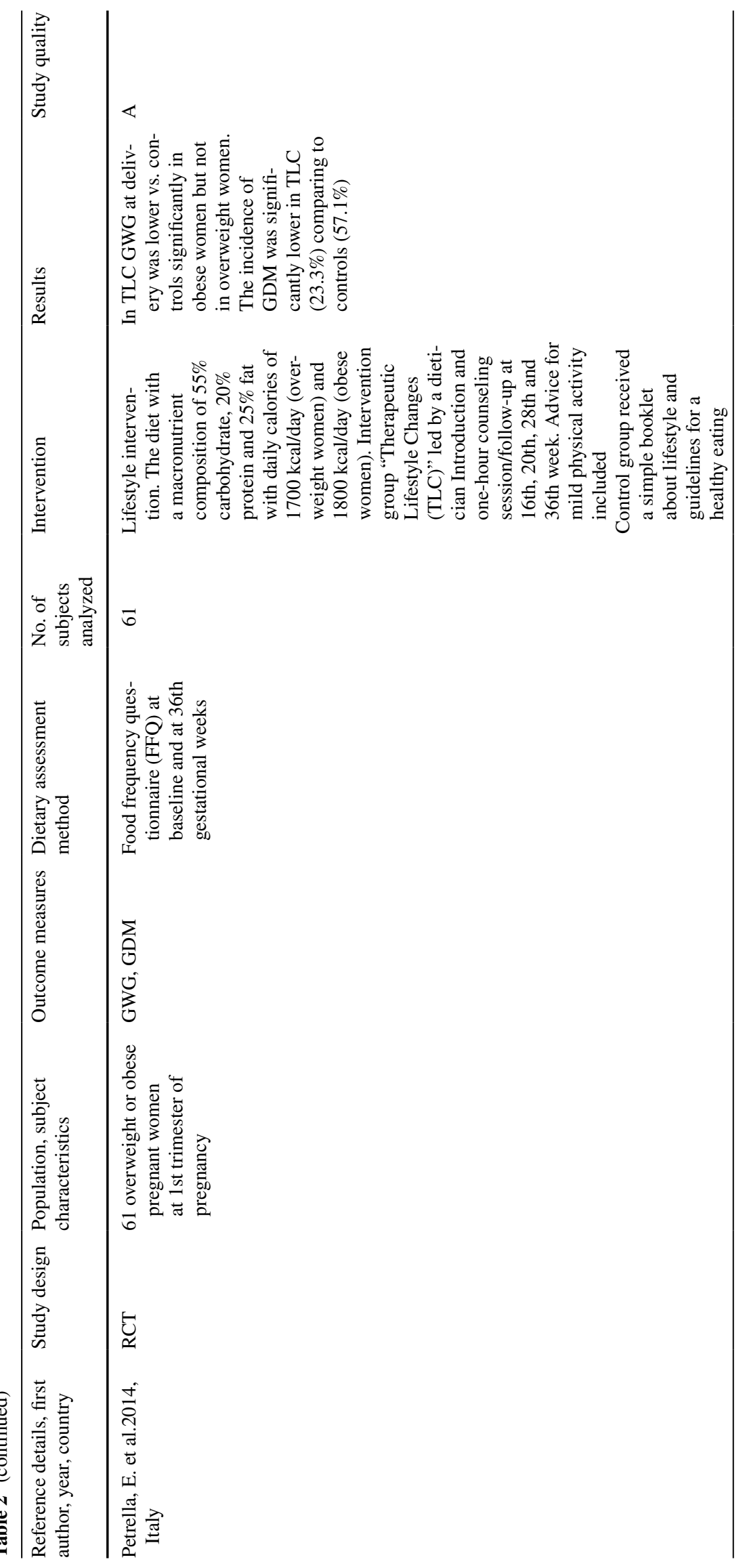




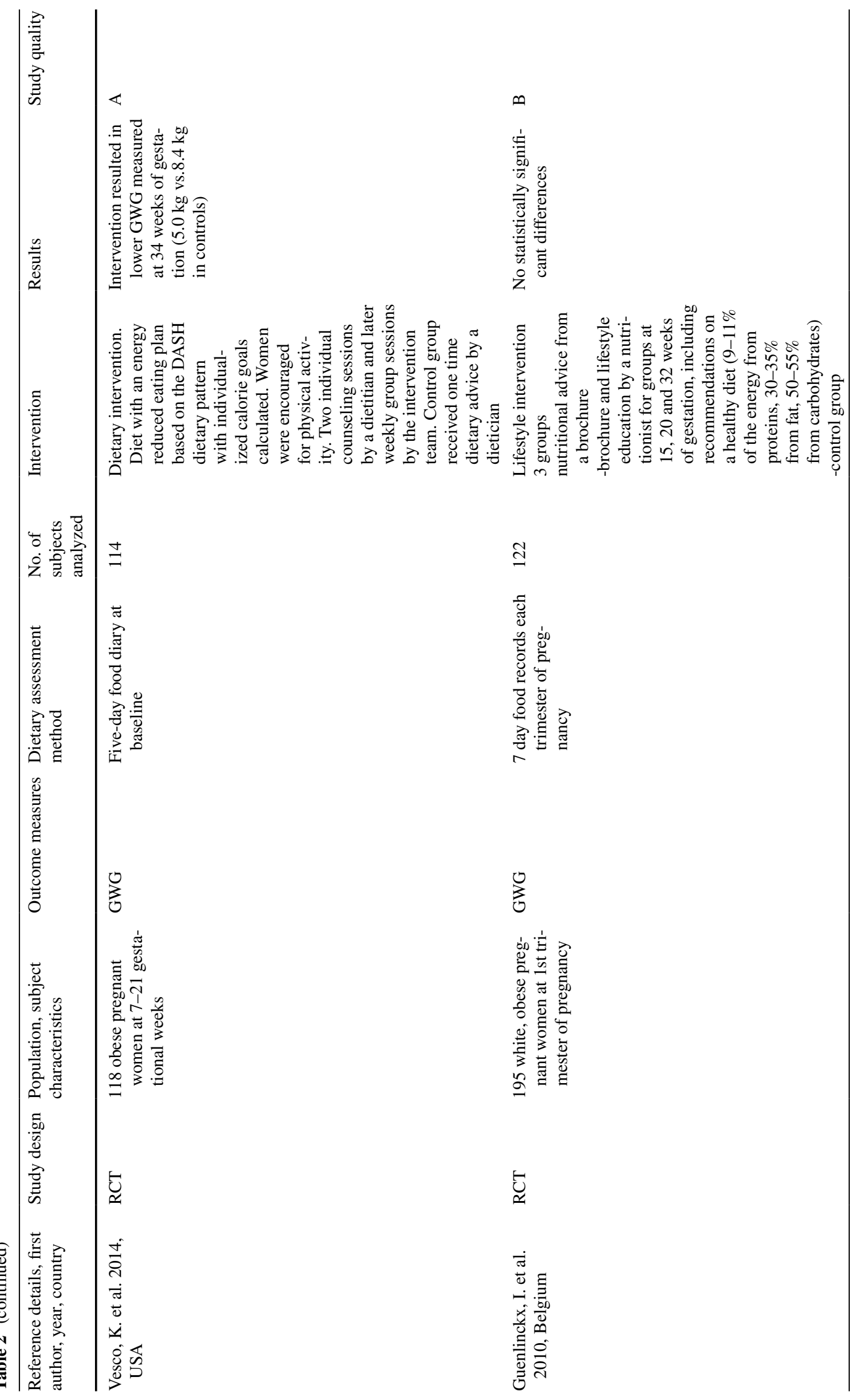




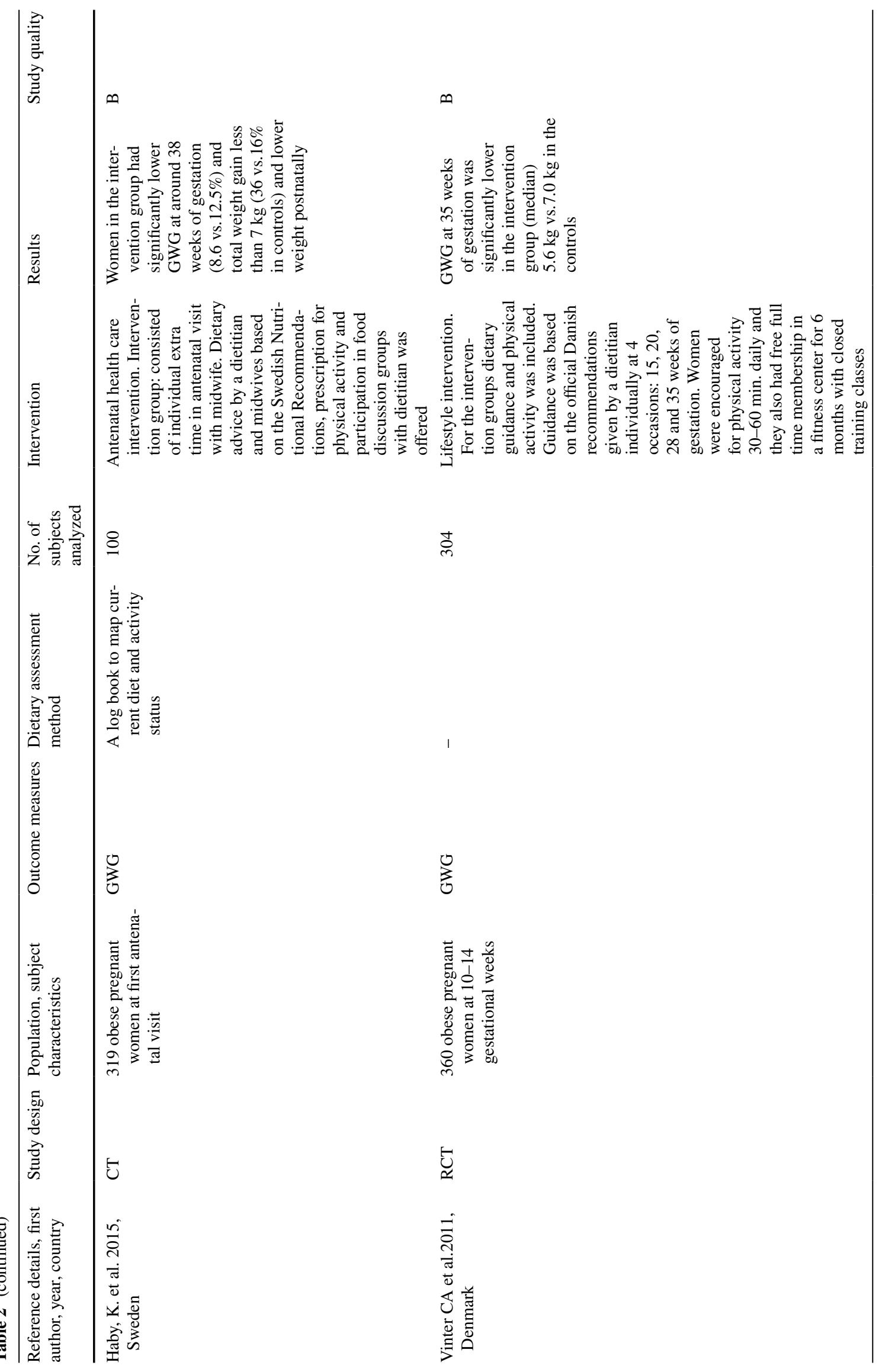




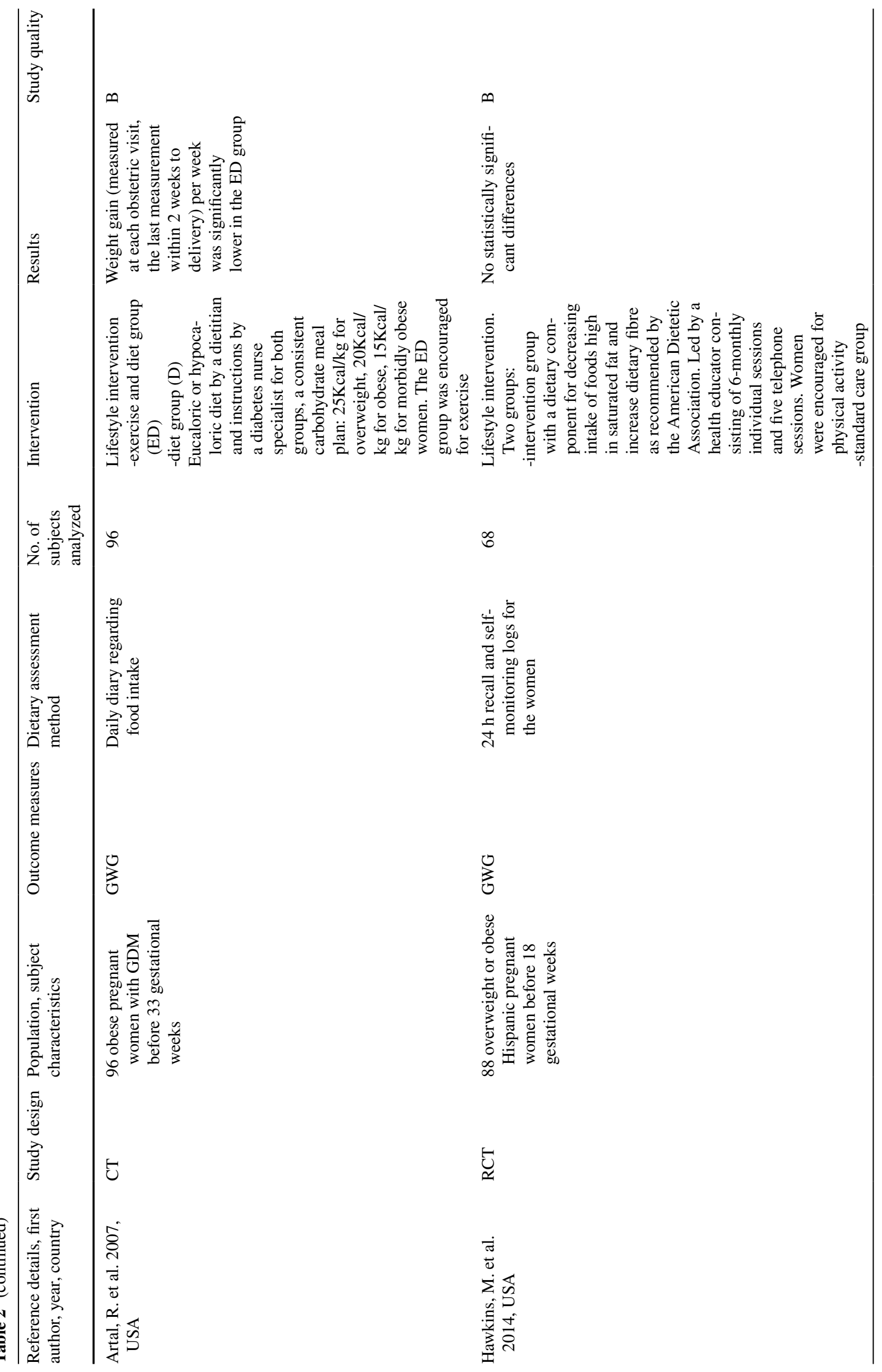




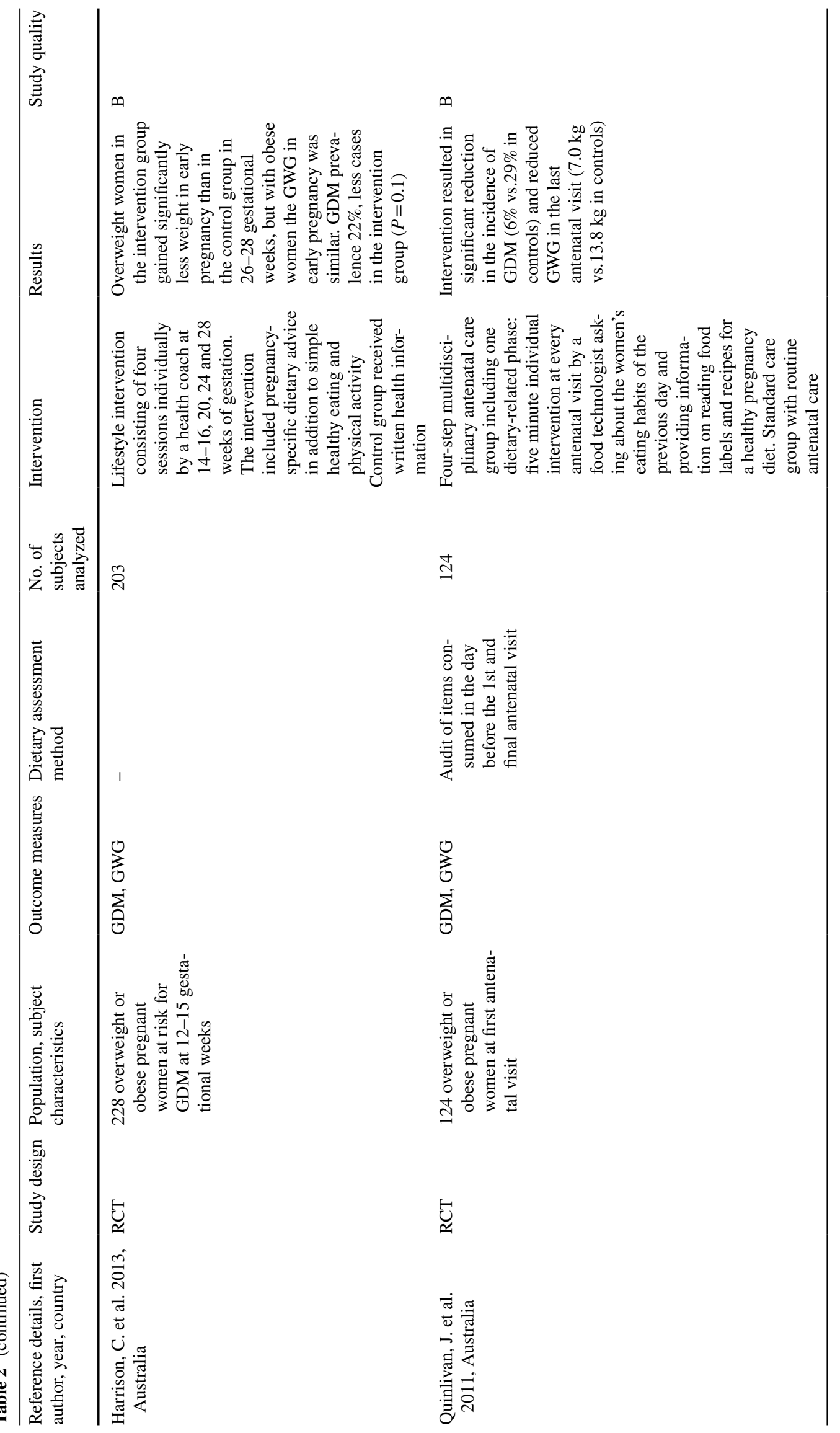




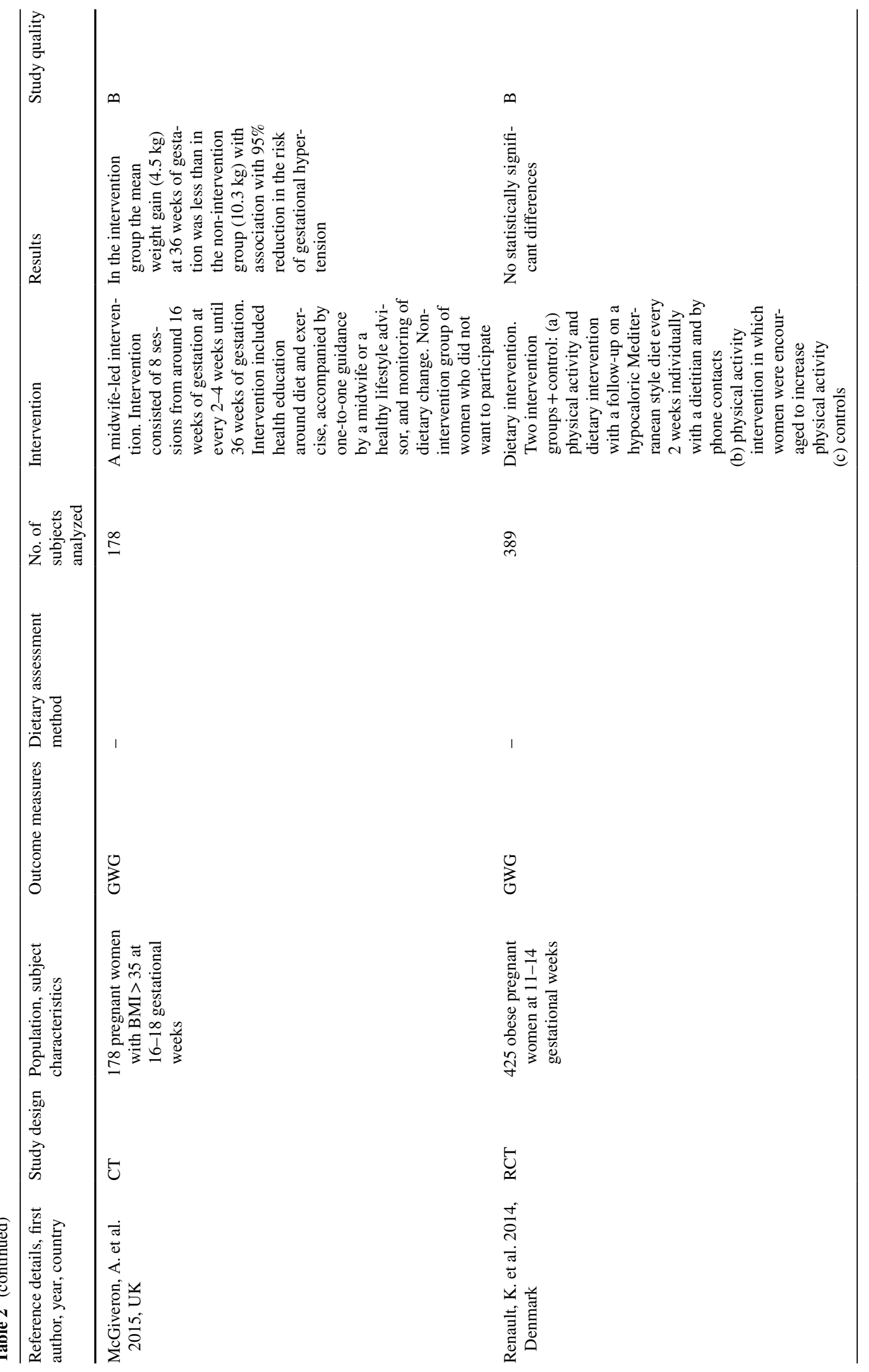


mainly delivered by a midwife or a lifestyle/ health coach had impacts on the outcomes. The roles of midwives or nurses in the interventions were highlighted in only four studies [12, 32, 36, 38], in which each was reported as effective. Three of these studies [12, 36, 38] included physical activity, in addition to dietary advice or a specified diet.

In the five studies reporting no significant differences between the intervention and control groups, the study designs were similar to those in which the intervention was found to be effective. However, two of the non-effective interventions did not include a specified diet [29,37], but dietary advice was included, and two studies did not include physical activity [30,34]. There were no differences in the efficacy based on the time of recruiting the women in the intervention. One study reported that the interventions were effective with overweight women but not obese women [27], and another study reported the opposite results [28]. Other studies did not report any significant differences when participants were stratified by BMI category as overweight vs. obese women. In most of the studies, GWG was measured at the end of the pregnancy between gestational week 38 and the time of delivery $[12,26,28,32,34,36]$, and in three studies, GWG was measured at approximately 36 weeks of gestation $[31,38,39]$. In the remaining studies, the time of the GWG measurement varied from 26 to 35 weeks of gestation.

\section{Discussion}

\section{Reflection on the results}

This systematic review confirms the potential of limiting the GWG or preventing GDM through different dietary interventions in overweight or obese pregnant women since the majority of the studies reported significant differences between the study and control groups. However, this review also confirms the variability in the strategies used to deliver dietary interventions in studies aiming to limit GWG and prevent GDM and, hence, the difficulty of summarizing the components of effective dietary interventions [22]. Dietary interventions were selected for this review because they have been previously reported to be the most effective type of interventions for limiting the GWG and preventing GDM [21].

Although the majority of the selected studies reported the interventions to be effective, there still appears to be inconsistency in the results since there were five studies that reported no significant differences between the groups but consisted of similar components as those studies that reported that the interventions were effective. The time of measuring the GWG varied from as early as 26 weeks of gestation until the day of delivery, but in those two studies reporting GWG at approximately 26 weeks of gestation [27, 29], the GWG was not either the main outcome measure or titled as "GWG in early pregnancy". It appears to be difficult to note other specific weaknesses in the studies in relation to the study design, participants or delivery of the dietary intervention.

Despite limiting this systematic review to studies including a dietary-intervention and the assessed outcome of the studies including either GWG or GDM, there still appears to be much variation in the dietary approach within the studies. Some studies focused on a specified diet with limited amounts of nutrients or energy and others used strategies of delivering dietary advice or counselling based on National Recommendations. A certain extent of physical activity was also included in most of the studies. The impact of the varying dietary approaches on GWG and GDM remain unclear because the interventions consisted of different components with additional variation in the implementation of the components, such as individual vs. group-based counselling.

In a review by Heslehurst and colleagues [40] it was stated that most of the intervention studies related to supporting weight management during pregnancy have focused on changing the pregnant women's behaviour. These studies have not focused on encouraging public health nurses (PHN) or other health professionals, such as midwives, to implement weight management guidelines into practice and provide counselling related to these issues. These practices could be important in motivating women. The commitment to the lifestyle changes required by the interventions is often difficult to achieve [40, 41]. It has been suggested that midwives should be offered support and education to keep up to date with the guidance on weight management and behaviour change techniques during pregnancy because they play a key role in health promotion [15].

Interestingly, in this systematic review, the midwives' or nurses' role in the interventions was mentioned in a minority of the studies. In addition, dietary advice was less often focused on groups, which could offer plenty of advantages both at the organizational and individual level since it is more cost-effective and offers peer-support for the women, which can have a positive effect as well on compliance with the intervention.

The quality of the studies assessed by the AHRQ Methods Reference Guide for Effectiveness and Comparative Effectiveness Reviews quality assessment tool [24] was generally good, and there were no differences in the efficacy of the interventions in relation to the assessed quality of the study regardless of whether it was graded as A or B. The AHRQ quality assessment tool is tailored for evaluating studies with dietary-interventions. In this review, not all of the selected studies included an actual dietary-intervention, but a dietary component with advice or counselling without detailed information was included; thus, the quality assessment tool 
was not entirely applicable to all of the studies. This may have some effect on the grading results.

Most of the studies $(n=13)$ selected in this systematic review were conducted in the year 2010 and beyond. This probably reflects the fact that overweightness and obesity are increasing health concerns in the obstetric population, and there is a need to develop interventions that could impact the weight management and health of the pregnant women and their newborns.

In future research it would be crucial to tailor the duration and contents of dietary interventions more consistently, for example based on national nutritional recommendations. More importance should be placed as well on the provider of the dietary intervention to allow for both the dietitian and the midwife or the public health nurse in the antenatal care to be deeply involved in the intervention. This could enable a smooth transition of the experiences from trials to every day practice in maternal care, which could also improve the quality of antenatal dietary counselling targeted for overweight and obese pregnant women.

\section{Limitations of the study}

A limitation of this study is the exclusion of studies written in languages other than English, which may cause a minor publication bias. The exclusion of pilot studies may have slightly reduced the number of studies included in this review; however, this exclusion is related to the quality of the review. The search did not encompass grey literature (e.g., theses, conference proceedings, etc.), which may cause some bias in the results of this review.

In the search and the selection of the articles for this review the behaviour change technique (BCT) taxonomy was not used to help in categorising the interventions or components used in the studies, which could have provided clearer results in this review [42]. Instead, each study was carefully screened to determine the type and components of the dietary intervention used.

\section{Financial support}

This study has been financially supported by the Finnish Foundation for Nurse Education and the Finnish Cultural Foundation for RL as research grants.

Author contribution R.L., K.V-J and U.S participated in the design of the study. R.L performed the literature search, and K.V-J and U.S participated in the selection of the studies. R.L, K.V-J and U.S performed the quality assessment of the studies. R.L reviewed the literature and wrote the manuscript with contributions by K.V-J and U.S. All authors read and approved the final manuscript.

\section{Compliance with ethical standards}

Conflict of interest All authors declare that they have no conflict of interest.

Open Access This article is distributed under the terms of the Creative Commons Attribution 4.0 International License (http://creativecommons.org/licenses/by/4.0/), which permits unrestricted use, distribution, and reproduction in any medium, provided you give appropriate credit to the original author(s) and the source, provide a link to the Creative Commons license, and indicate if changes were made.

\section{References}

1. WHO 2014. http://www.who.int/topics/obesity/en/. Accessed April 2016

2. Euro-Peristat 2010, The European Perinatal Health Report. http://www.europeristat.com/reports/european-perinatal-healthreport-2010.html. Accessed Dec 2017

3. THL 2016. Perinataalitilasto-synnyttäjät, synnytykset ja vastasyntyneet 2015. http://www.julkari.fi/handle/10024/131259. Accessed Nov 2016

4. Catalano P (2010) Obesity. In: Queenan JT, Hobbins JC, Spong CY (eds) Protocols for high-risk pregnancies: an evidence-based approach, 5th edn. Blackwell Science Ltd., pp 223-226

5. Mission J, Marshall N, Caughey A (2013) Obesity in pregnancy: a big problem and getting bigger. Obstet Cynecol Surv 68(5):389-399

6. Lamminpää R, Vehviläinen-Julkunen K, Gissler M, Selander T, Heinonen S (2016) Pregnancy outcomes of overweight and obese women aged 35 years or older-a registry-based study in Finland. Obesity Res Clin Pract 10(2):133-142

7. Heude B, Thiébaugeorges O, Goua V et al (2012) Pre-pregnancy Body Mass Index and Weight Gain During Pregnancy: Relations with Gestational Diabetes and Hypertention, and Birth Outcomes. Matern Child Health J 16:355-363

8. Ehrenberg H, Dierker L, Milluzzi C, Mercer B (2002) Prevalence of maternal obesity in an urban center. Am J Obstet Gynecol 187(5) 1189-1193

9. Catalano P, Ehrenberg H (2006) The short- and long-term implications of maternal obesity on the mother and her offspring. BJOG113:1126-1133

10. Guo L, Liu J, Ye R et al (2015) Gestational weight gain and overweight in children 3-6 Years. J Epidemiol 25(8):536-543

11. IOM 2009 Weight-gain during pregnancy. http://iom.edu/ / media/Files/Report\%20Files/2009/Weight-Gain-During-Pregnancy-Reexamining-the-Guidelines/Report\%20Brief\%20-\%20 Weight\%20Gain\%20During\%20Pregnancy.pdf. Accessed Oct 2016

12. Haby K, Glantz A, Hanas R et al (2011) Mighty mums-an antenatal health care intervention can reduce gestational weight gain in women with obesity. Midwifery 31:685-692

13. Einerson B, Hufman J, Istwan N et al (2011) New gestatational weight gain guidelines: an observational study of pregnancy outcomes in obese women. Obesity 19(12):2361-2364

14. Jebeile H, Mijatovic J, Louie J et al (2016) A systematic review and metaanalysis of energy intake and weight gain in pregnancy. Am J Obstet Gynecol 214(4):465-483

15. Martin S, Duxbury A, Soltani H (2014) An overview of evidence on diet and physical activity based interventions for gestational weight management. Evidence Based Midwifery 12(2):40-45 
16. Ruifrok A, van Poppel M, van Wely M et al (2014) Association between weight gain during pregnancy and pregnancy outcomes after dietary and lifestyle interventions: a meta-analysis. Am J Perinatol 31(5):353-363

17. Elliot-Sale K, Barnett J, Sale C (2015) Exercise interventions for weight management during pregnancy and up to 1 year postpartum among normal weight, overweight and obese women: a systematic review and meta-analysis. Br J Sports Med 49(20):1-8

18. Ronnberg AK, Nilsson K (2010) Interventions during pregnancy to reduce excessive gestational weight gain: a systematic review assessing current clinical evidence using the Grading of Recommendations, Assessment, Development and Evaluation (GRADE) system. BJOG 117(11):1327-1334

19. Oostdam K, van Poppel M, Wouters M et al (2011) Interventions for preventing gestational diabetes mellitus: a systematic review and meta-analysis. J Women's Health 20(10):1551-1563

20. Tieu J, Crowther CA, Middleton P (2008) Dietary advice in pregnancy for preventing gestational diabetes mellitus. Cochrane database of systematic reviews

21. Flynn AC, Dalrymple K, Barr S et al (2016) Dietary interventions in overweight and obese pregnant women: a systematic review on the content, delivery and outcomes of randomized controlled trials. Nutr Rev 74(5):312-328

22. Thangaratinam $\mathrm{T}$ et al (2012) Effects of interventions in pregnancy on maternal weight and obstetric outcomes: meta-analysis of randomised evidence. BMJ 344:e2088

23. Rogozinska E, D’Amico MI, Khan KS et al (2016) Development of composite outcomes for individual patient data (IPD) metaanalysis on the effects of diet and lifestyle in pregnancy: a Delphi survey. BJOG 123(2):190-198

24. A guide for conducting Systematic Literature Reviews for the 5th edition of the Nordic Nutrition Recommendations

25. Catanzaro M (1988) Using qualitative analytical techniques. In: Woods P, Catanzaro M (eds) Nursing research: theory and practice. C. V. Mosby Company, New York, NY, pp 437-456

26. Quinlivan J, Lam T, Fisher J (2011) A Randomized trial of a four-step multidisciplinary approach to the antenatal care of obese pregnant women. Aust NZ J Obstet Gynaecol 51:141-146

27. Harrison C, Lombard C, Strauss B et al (2013) Optimizing healthy gestational weight gain in women at high risk of gestational diabetes: a randomized controlled trial. Obesity 21(5):904-909

28. Petrella E, Malavolti M, Bertarini V et al (2014) Gestational weight gain in overweight and obese women enrolled in a healthy lifestyle and eating habits program. J Matern-Fetal Neonatal Med 27(13):1348-1352

29. Poston L, Bell R, Croker H et al (2015) Effect of a behavioural intervention in obese pregnant women (the UPBEAT study): a multicentre, randomized controlled trial. Lancet 3:767-777

30. Markovic T, Muirhead R, Overs S et al (2016) Randomized controlled trial investigating the effects of a low-glycemic index diet on pregnancy outcomes in women at high risk of gestational diabetes mellitus: the GI Baby 3 study. Diabetes Care 39:31-38

31. Wolff S, Legarth J, Vangsgaard K et al (2008) A Randomised trial of the effects of dietary counseling on gestational weight gain and glucose metabolism in obese women. Int J Obesity 32:495-501

32. Bogaerts AFL, Devlieger R, Nuyts E et al (2013) Effects of lifestyle intervention in obese pregnant women on gestational weight gain and mental health: a randomized controlled trial. Int J Obesity $37: 814-821$

33. Vesco K, Karanja N, King J et al (2014) Efficacy of a group-based dietary intervention for limiting gestational weight gain among obese women:a randomized trial. Obesity 22(9):1989-1996

34. Guenlinckx I, Devlieger R, Mullie P et al (2010) Effect of lifestyle intervention on dietary habits, physical acticity, and gestational weight gain in obese pregnant women: a randomized controlled trial. Am J Clin Nutr 91:373-380

35. Vinter C, Jensen D, Ovesen P et al (2011) The LiP (Lifestyle in Pregnancy) Study. A randomized controlled trial of lifestyle intervention in 360 obese pregnant women. Diabetes Care 34:2502-2504

36. Artal R, Catanzaro R, Gavard J et al (2007) A lifestyle intervention of weight-gain restriction: diet and exercise in obese women with gestational diabetes mellitus. Appl Physiol Nutr Metab 32:596-601

37. Hawkins M, Hosker M, Marcus BH et al (2014) A pregnancy lifestyle intervention to prevent gestational diabetes risk factors in overweight Hispanic women: a feasibility randomized controlled trial. Diabet Med 32:108-115

38. McGiveron A, Foster S, Pearce J et al (2014) Limiting antenatal weight gain improves maternal health outcomes in severely obese pregnant women: findings of a pragmatic evaluation of a midwifeled intervention. J Hum Nutr Diet 28(Suppl 1):29-37

39. Renault K, Norgaard K, Nilas L et al (2014) The treatment of obese pregnant women (TOP) study: a randomized controlled trial of the effect of physical activity intervention assessed by pedometer with or without dietary intervention in obese pregnant women. AJOG 134e1-134e9

40. Heslehurst $\mathrm{N}$, Crowe $\mathrm{L}$, Robalino $\mathrm{S}$ et al (2014) Interventions to change maternity healthcare professionals' behaviours to promote weight-related support for obese pregnant women: a systematic review. Implement Sci 9:97

41. Dennedy M (2010) The maternal and fetal impacts of obesity and gestational diabetes on pregnancy outcome. Best Practi Res Clin Endocrinol Metabol 24:573-589

42. Michie S, Carey R, Johnston M et al (2016) From theory-inspired to theory-based interventions: a protocol for developing and testing a methodology for linking behaviour change techniques to theoretical mechanisms of action. Ann Behav Med. doi:10.1007/ s12160-016-9816-6 